\title{
Le fratture del collo femore
}

\author{
Nicola Pace \\ UO Ortopedia, Ospedali Riuniti Murri-Jesi, Via della Vittoria 76, 60035, Jesi (AN), Italia \\ nicopace@yahoo.com
}

Pubblicato online: 9 luglio 2013

(C) Springer-Verlag Italia 2013

Questo numero monografico ha voluto deliberatamente differenziarsi rispetto ai precedenti cercando di esplorare nuove possibilità di reperire risorse scientifiche per la crescita della rivista.

Infatti esso raccoglie esperienze, studi monotematici affidati a esperti del settore reclutati dall'autore, il quale si è dedicato esclusivamente alla parte organizzativa chiamandosi fuori da quella compilativa.

E stato scelto come tema le fratture del collo del femore, un argomento apparentemente banale e da alcuni definibile ormai come totalmente esplorato, quasi decantato.

$\mathrm{Ma}$ le fratture che giungono quotidianamente nei reparti ortopedici della medicina ospedaliera pubblica aumentano continuamente di numero, aumentano di difficoltà per l'età sempre più avanzata dei pazienti, per l'associazione con comorbilità sempre maggiori, per una organizzazione ospedaliera sempre più precaria.

Tutto questo comporta un aumento di difficoltà, un aumento di responsabilità, una necessità di aggiornamento continuo, scientifico e organizzativo, che solo attraverso la riproposizione di questo argomento può essere soddisfatta. Per questo motivo si è voluto inquadrare l'argomento in tre gruppi: quello delle scelte tecniche operative con le indicazioni e i risultati, quello dell'analisi delle possibili complicanze, che non rappresentano sempre errori di indicazione o errori di tecnica operatoria, ma sempre più spesso alterate risposte biologiche dell'individuo ai due insulti traumatici rappresentati dalla frattura in sé e dall'infissione attraverso un atto chirurgico di materiale estraneo all'organismo, che necessita almeno di una compatibilità biologica con l'indi- viduo ospitante. Per ultimo si è inserito il concetto di consenso informato, conformandolo alle fratture del femore e al "paziente" fratturato; è stato considerato con il termine di paziente la persona affetta con tutte le sue problematiche, spesso anche psichiche e sociali, e anche la famiglia o la casa protetta da cui proviene, perché per ottenere una condivisione di trattamento chirurgico e riabilitativo è obbligatorio conoscere e responsabilizzare tutto il contesto che ruota intorno al paziente anziano fratturato.

Voglio infine ringraziare il Dott. Francesco Brutto e Biomet Italia per il supporto economico che hanno concesso a $L o$ Scalpello Otodi Educational, contributo che rappresenta un segno di vicinanza e di stima tra mondo dei device e mondo scientifico: un rapporto difficile ma possibile, da mantenere anche in questo momento di fortissima crisi economica.

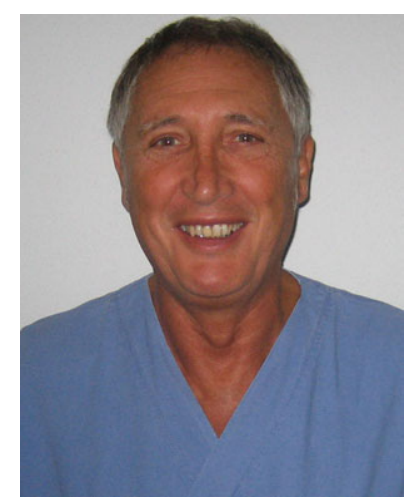

